\title{
SARS-CoV-2-related Progressive Brain White Matter Lesion Associated with an Increased Cerebrospinal Fluid Level of IL-6
}

\author{
Yuwa Oka ${ }^{1}$, Akihiro Ueda ${ }^{1}$, Tomokazu Nakagawa ${ }^{1}$, Yujiro Kikuchi ${ }^{2}$, Daiki Inoue ${ }^{2}$, \\ Satoshi Marumo ${ }^{2}$ and Sadayuki Matsumoto ${ }^{1}$
}

\begin{abstract}
:
We herein report a 49-year-old man with a fever, diagnosed with severe acute respiratory syndrome coronavirus 2 (SARS-CoV-2) infection. After two weeks of hospitalization, he suddenly mentioned visual field impairment. Computed tomography and magnetic resonance imaging revealed white matter damage and vasogenic edema. Cerebrospinal fluid showed increased levels of interleukin (IL)-6. His symptoms and white matter lesion deteriorated. After treatment with intravenous methylprednisolone therapy and plasmapheresis, his symptoms and white matter lesion improved gradually. We suspect that our patient was affected by a secondary hyperinflammatory syndrome related to cytokines, alone or in combination with direct viral injury through endothelial cell damage. The IL-6 levels were elevated only in the cerebrospinal fluid, suggesting focal central nervous system inflammation.
\end{abstract}

Key words: COVID-19, SARS-CoV-2, IL-6, CSF, CNS dysfunction

(Intern Med 60: 3167-3170, 2021)

(DOI: 10.2169/internalmedicine.8123-21)

\section{Introduction}

The severe acute respiratory syndrome coronavirus 2 (SARS-CoV-2) has caused a global pandemic, presenting primarily with respiratory symptoms. Various neurological complications have also been reported following SARSCoV-2 infection (1-3). We herein report a case of progressing white matter damage after SARS-CoV-2 infection with a response to plasmapheresis. Brain magnetic resonance imaging (MRI) findings and cerebrospinal fluid (CSF) were evaluated through treatment.

\section{Case Report}

A 49-year-old man was admitted to our hospital with a high fever and throat pain. The patient had a fever for three days before arriving at the hospital. Polymerase chain reaction (PCR) of nasopharyngeal swab specimens tested posi- tive for SARS-CoV-2. His medical history indicated epilepsy, for which the patient was taking anti-epileptic medications (phenytoin $200 \mathrm{mg} /$ day, levetiracetam $375 \mathrm{mg} /$ day, perampanel $4 \mathrm{mg} /$ day and zonisamide $100 \mathrm{mg} /$ day). On admission to our hospital (day 1), the patient's consciousness, hemodynamics, and respiration were stable. Chest radiography revealed scattered ground-glass opacity in the bilateral lung fields. We treated the patient with oral corticosteroids, antibiotics, anticoagulants, and supportive care.

Day 13 PCR of a nasopharyngeal swab taken from the patient was negative for SARS-CoV-2. The disease progressed well until the patient mentioned visual field impairment on day 15. A visual field examination found left homonymous hemianopia. No other neurological abnormalities were present. Brain computed tomography (CT) showed low-density areas in the white matter of the right cerebral hemisphere (Fig. 1A). Brain T2-weighted imaging (T2WI) and fluid-attenuated inversion-recovery (FLAIR) MRI showed a high intensity at the white matter of the right cere-

${ }^{1}$ Department of Neurology, Kitano Hospital, Tazuke Kofukai Medical Research Institute, Japan and ${ }^{2}$ Respiratory Disease Center, Kitano Hospital, Tazuke Kofukai Medical Research Institute, Japan 

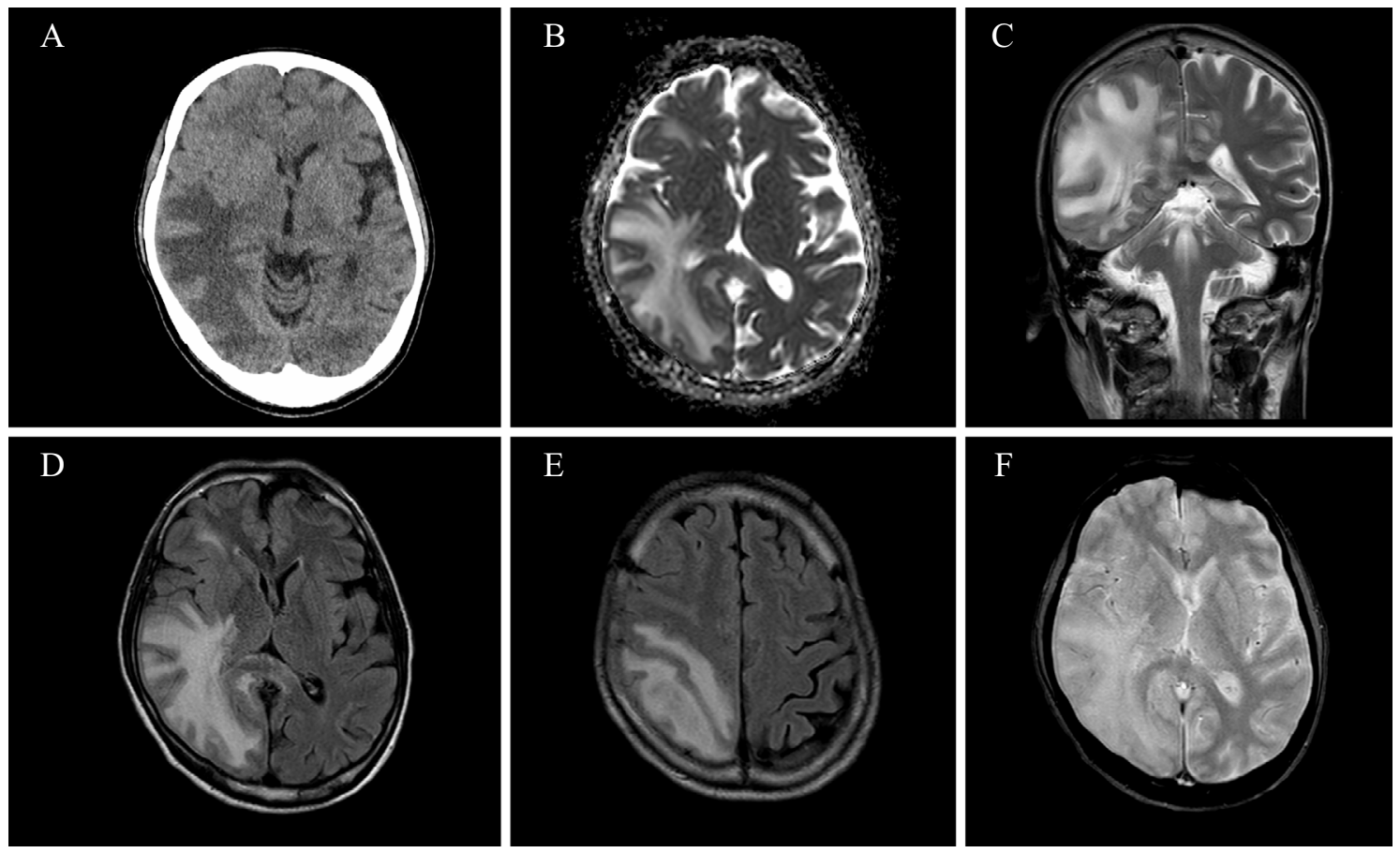

Figure 1. Brain CT at day 14 (A). CT showed low-density areas at the white matter of the right cerebral hemisphere. Brain MRI at day 15 (B-F). White matter of the right cerebral hemisphere showed hyperintensity on apparent diffusion coefficient (ADC) values in diffusion-weighted imaging (B). Coronal T2WI (C) and axial FLAIR (D, E) showed high intensity at the white matter of the right cerebral hemisphere, including the corpus callosum (C, D, E). T2*-weighted imaging showed no abnormalities (F).

bral hemisphere, including the corpus callosum (Fig. 1C-E). The same area showed hyperintensity on apparent diffusion coefficient values in diffusion-weighted imaging, indicating vasogenic edema (Fig. 1B). Inflammation of the white matter and brain edema were suspected. Magnetic resonance angiography appeared normal. T2*-weighted imaging showed no signs of cerebral microbleeds (Fig. 1F).

We administered intravenous methylprednisolone therapy (1,000 mg/day) for 3 days, followed by oral prednisolone maintenance therapy during days 15 to 19 . A CSF study on day 16 showed a leukocyte count of $136 / \mu \mathrm{L}(40.4 \%$ polymorphonuclear neutrophils), protein level of $129.2 \mathrm{mg} / \mathrm{dL}$, interleukin (IL)-6 at 1,750 pg/mL (blood IL-6 level was 2.4 $\mathrm{pg} / \mathrm{mL}$ ), and glucose level of $68 \mathrm{mg} / \mathrm{dL}$ (corresponding blood glucose level was $138 \mathrm{mg} / \mathrm{dL}$ ). The $\mathrm{IgG}$ index was increased (0.9). Oligoclonal bands were negative in the CSF and serum. Serum tested negative for myelin oligodendrocyte glycoprotein (MOG)-antibody in subsequent cell-based assays. Quantitative PCR did not detect SARS-CoV-2 in the CSF.

A day 19 examination found drooping of the left mouth corner and left hemiparalysis that left him unable to walk. MRI showed expansion to the frontal lobe and worsening of brain edema. We treated the patient with intravenous methylprednisolone for three more days, but follow-up MRI on day 21 showed worsening brain edema and lesion expansion (Fig. 2A, B). By this time, the patient needed time to answer questions but was oriented in time, place, and person.
Plasma exchange was initiated on day 22.

The patient communicated in an orderly manner, and the mouth droop and hemiparalysis showed rapid recovery after plasmapheresis treatment, but the left homonymous hemianopia persisted. A follow-up CSF study on day 33 showed considerable improvement (leukocyte count, $11 / \mu \mathrm{L}$; protein level, $52.4 \mathrm{mg} / \mathrm{dL}$; IL-6, $2.3 \mathrm{pg} / \mathrm{mL}$ ). Follow-up MRI on day 40 revealed improvement in the white matter lesion and brain swelling as well (Fig. 2C, D). Cerebral microbleeds were evident in the white matter of the right parietal and occipital lobes (Fig. 2E). Arterial spin-labeling (ASL) showed hypoperfusion of the affected area (Fig. 2F). At day 47, FLAIR showed further improvement (Fig. 2G, H). Following rehabilitation, he was able to walk again without any assistance. Left homonymous hemianopia did not fully recover but did show mild improvement.

\section{Discussion}

The state of our patient worsened progressively despite intravenous steroid treatment. However, combination with plasma exchange treatment managed to stop this deterioration, and his physical examination findings showed gradual improvement. CSF evaluations and MRI also demonstrated the therapeutic effect. SARS-CoV-2-associated central nervous system (CNS) damage was reported to be incurred through several mechanisms, including direct viral, inflammatory-mediated, thrombotic, and hypoxia-mediated 

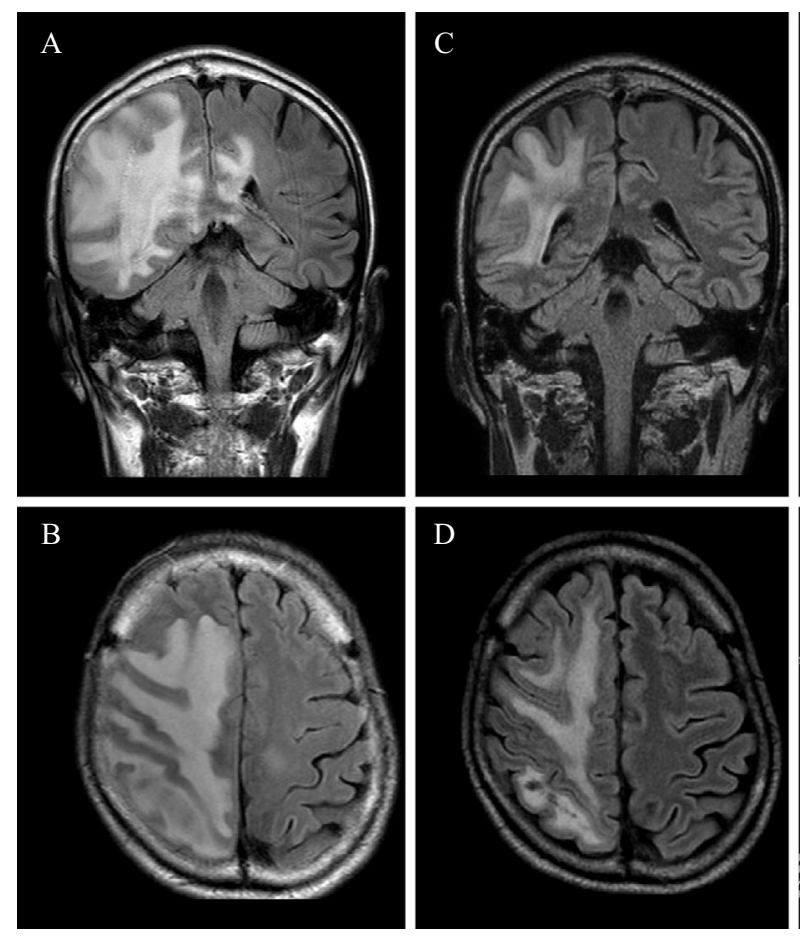
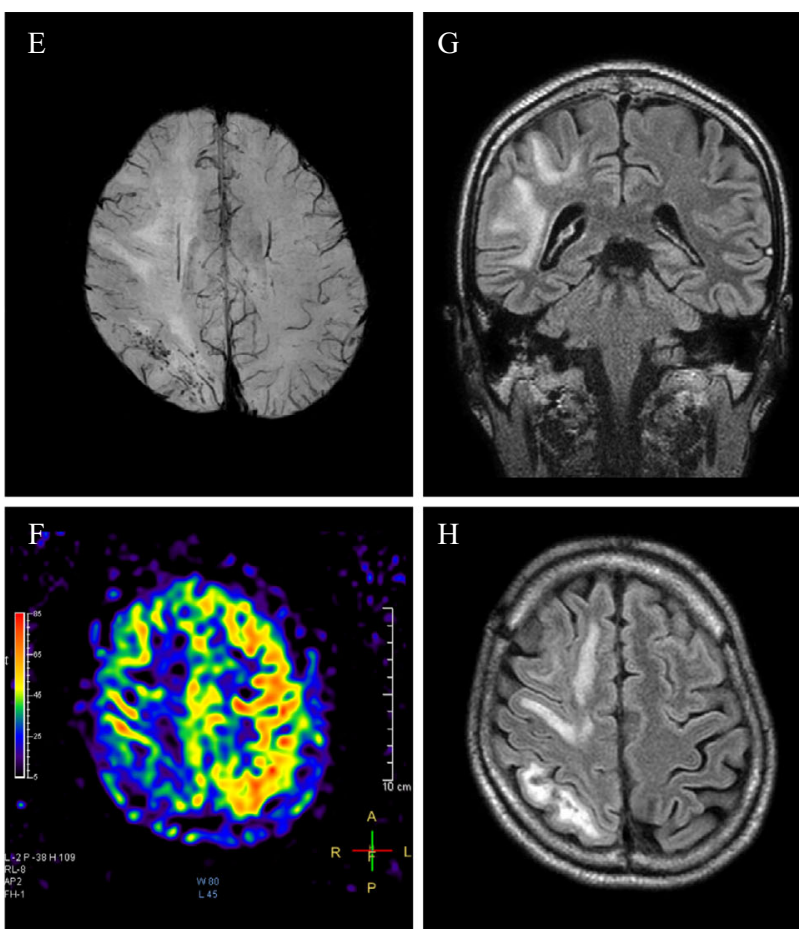

Figure 2. Coronal (A) and axial (B) FLAIR on day 21 showed expanded white matter lesion and worsened cerebral edema. Coronal (C) and axial (D) FLAIR after plasmapheresis (day 40). SWI showed cerebral microbleeds in the white matter of the right parietal and occipital lobes (E). ASL showed hypoperfusion of the right hemisphere, especially in the affected area (F). Coronal (G) and axial (H) FLAIR at day 47. The white matter lesion gradually resolved after plasmapheresis.

injuries (1). Since our patient reacted well to plasmapheresis, inflammatory-mediated injury was considered the primary cause. Activation of inflammatory pathways, such as IL-6, might cause SARS-CoV-2-associated encephalitis $(4,5)$. Since the CSF IL-6 level decreased after successful treatment, we suspected that the IL-6 effect was the primary cause of the white matter damage. Another study reported the possibility of cross-reactive antibodies generated in response to SARS-CoV-2 infection (6). Neurological symptoms in our case may have persisted because of antibody cross reactivity.

In addition, direct viral injury can also cause progressive white matter damage and brain edema $(1,5)$. A negative CSF PCR result may suggest that viruses have invaded the nervous system directly (7). It was reported that viral damage to the endothelial lining could facilitate viral access to the brain (8). The cerebral microbleeds in our patient (Fig. 2E) might have resulted from a direct viral injury. Microbleeds were not detected on initial T2*-weighted imaging (Fig. 1F). Focal inflammation and continuously expanding edema in the white matter also imply direct viral invasion. The hypoperfusion seen during ASL (Fig. 2F) suggests that hypoxia may have caused some damage as well.

In summary, we suspect that our patient was affected by a secondary hyperinflammatory syndrome related to cytokines, alone or in combination with direct viral injury through endothelial cell damage. The IL-6 levels were elevated only in the CSF, suggesting focal CNS inflammation. In our case, treatment with intravenous methylprednisolone along with plasmapheresis was effective. The therapeutic effect of plasma exchange in COVID-19-related encephalopathy has been reported previously (9). However, spontaneous remission should also be considered. Further studies are necessary to clarify our findings.

The authors state that they have no Conflict of Interest (COI).

\section{Acknowledgements}

The authors thank Dr. Takeshi Sawada, Department of Radiology, Kitano Hospital, Tazuke Kofukai Medical Research Institute, for his helpful comments. The authors thank Dr. Toshiyuki Takahashi, Tohoku University, for performing the measurements of anti-MOG antibody.

\section{References}

1. Bodro M, Compta Y, Sánchez-Valle R. Presentations and mechanisms of CNS disorders related to COVID-19. Neurol Neuroimmunol Neuroinflamm 8: e923, 2021.

2. Helms J, Kremer S, Merdji H, et al. Neurologic features in severe SARS-CoV-2 infection. New Engl J Med 382: 2268-2270, 2020.

3. Amruta N, Chastain WH, Paz M, et al. SARS-CoV-2 mediated neuroinflammation and the impact of COVID-19 in neurological disorders. Cytokine Growth Factor Rev 58: 1-15, 2021.

4. Bodro M, Compta Y, Llansó L, et al. Increased CSF levels of IL-1 B, IL-6, and ACE in SARS-CoV-2-associated encephalitis. Neurol Neuroimmunol Neuroinflamm 7: e821, 2020.

5. Mehta P, McAuley DF, Brown M, et al. COVID-19: consider cy- 
tokine storm syndromes and immunosuppression. Lancet 395: 1033-1034, 2020.

6. Kreye J, Reincke SM, Prüss H. Do cross-reactive antibodies cause neuropathology in COVID-19? Nat Rev Immunol 20: 645-646, 2020.

7. Novi G, Rossi T, Pedemonte E, et al. Acute disseminated encephalomyelitis after SARS-CoV-2 infection. Neurol Neuroimmunol Neuroinflamm 7: e797, 2020.

8. Baig AM, Khaleeq A, Ali U, Syeda H. Evidence of the COVID-19 virus targeting the CNS: tissue distribution, host-virus interaction, and proposed neurotropic mechanisms. ACS Chem Neurosci 11: 995-998, 2020.

9. Ranganathan C, Fusinski SD, Obeid IM, et al. Therapeutic plasma exchange for persistent encephalopathy associated with Covid-19. eNeurologicalSci 22: 100327, 2021.

The Internal Medicine is an Open Access journal distributed under the Creative Commons Attribution-NonCommercial-NoDerivatives 4.0 International License. To view the details of this license, please visit (https://creativecommons.org/licenses/ by-nc-nd/4.0/).

(C) 2021 The Japanese Society of Internal Medicine Intern Med 60: 3167-3170, 2021 